\title{
A new approach to prevent hazelnut callus browning by modification of sub-culture
}

\author{
M. RAEISPOUR SHIRAZI, S.A. RAHPEYMA*, and J. ZOLALA \\ Department of Agricultural Biotechnology, Shahid Bahonar University of Kerman, Kerman 76169-14111, Iran
}

\begin{abstract}
Tissue culture of hazelnut (Corylus avellana L.) represented the promising strategy for production of its valuable compound, paclitaxel, but one of the most important problems is initial callus browning in its callus culture. To obtain healthy callus cells of $C$. avellana, three different culture media, Murashige and Skoog (MS), Nas and Read (NRM), and Driver and Koniuki (DKW), in combination with $500 \mathrm{mg} \mathrm{dm}^{-3}$ citric acid, $500 \mathrm{mg} \mathrm{dm}^{-3}$ acetic acid, and $500 \mathrm{mg} \mathrm{dm}^{-3}$ polyvinylpyrrolidone (PVP) were applied. These cultures were passed two sub-cultures in the same media. Also, a novel modified sub-culture system was designed and compared to routine techniques. The sub-culture system was changed as following: the induced calli were transferred to a liquid media with the same composition and after developing the cell suspension, the cells were immobilized on a solid medium. In the first experiment, it was demonstrated that the medium compositions had an effect on the growth rate and callus browning reduction although they could not eliminate the browning. The results have shown that the highest growth indices were related to NRM with $500 \mathrm{mg} \mathrm{dm}^{-3} \mathrm{acetic}^{\mathrm{acid}}$ $+500 \mathrm{mg} \mathrm{dm}^{-3}$ citric acid and to NRM with $500 \mathrm{mg} \mathrm{dm}^{-3}$ PVP in the first culture. These media had the least amount of browning $(9.15 \%)$. In the two next sub-cultures, NRM without additives had the maximum growth rate and the lowest browning was observed on the same media as in the first subculture $(13.3 \%)$. The technique of modified sub-cultures increases growth rate ten times in comparison with routine cultivation method.
\end{abstract}

Additional key words: ascorbic acid, citric acid, Corylus avellana L., in vitro culture, polyvinylpyrrolidone.

Hazelnut (Corylus avellana L.) from Betulaceae family is distributed in temperate regions of the world (Cronquist and Takhtadzhian 1981, Molnar 2011, Qaderi et al. 2012). It is the sixth commercial nut crops behind cashews (Anacardium occidentale L.), walnuts (Juglans regia L.), chestnuts (Castanea spp.), almonds [Prunus dulcis (Miller) D.A. Webb], and pistachio (Pistacia vera). Detection of paclitaxel (PC), an impressive anticancer agent, in hazelnut tissues, brought up it as a valuable medicinal plant and increased paying attentions to it (Hoffman et al. 1998, Hoffman and Shahidi 2009). The PC was initially isolated from Taxus brevifolia (Hoffman et al. 1998). However, commercial production of PC from Taxus sp. is going to be more sophisticated due to the increasing demand of PC from slow growing yew tree with a reducing population (Bedi et al. 1996, Abbasin et al. 2010). The hazelnut cell cultures have been proved to be effective alternative method for the production of secondary metabolites (Hussain et al. 2012). This method has advantages like modification of biosynthetic pathways in order to produce a high amount of secondary metabolites (Georgiev et al. 2009). The previous studies have shown that in vitro plant cell culture achieved from various tissues of hazelnut is one of the most promising approaches to provide a sustainable source of PC (Bestoso et al. 2006, Miele et al. 2012). Calli that are obtained from in vitro cultures of hazelnut seeds remain white and friable approximately over two years with regular sub-culture. These calli appear very soon (after several days), in media supplemented with callus inducing factors and their rapid growth compensates the less amount of PC in the hazelnut in comparison with yew callus which grow slower and stay in yellow pale and friable for less than one year (Bestoso et al. 2006, Miele et al. 2012). Production of PC in hazelnut cell suspension has been evaluated in different studies and its potential for commercial production of

Submitted 15 April 2019, last revision 16 January 2020, accepted 18 January 2020.

Abbreviations: AA - ascorbic acid; CA - citric acid; DKW - Driver and Koniuki medium; MS - Murashige and Skoog medium; NRM - Nas and Read medium; PC - paclitaxel; PVP - polyvinylpyrrolidone; RGR - relative growth rate.

* Corresponding author; e-mail: s.rahpeyma@uk.ac.ir 
PC is agreed (Gallego et al. 2017, Rahpeyma et al. 2017, Salehi et al. 2018). However, the biggest problem is its initial browning. Secondary metabolites like phenolic compounds are responsible for tissue browning in C. avellana and other plants during the establishment stage (Sugano et al. 1975, Yu and Reed 1995). Cell browning is the result of the oxidation of phenolic compounds (Khosroushahi et al. 2011) and the accumulation of these products. This phenomenon prevents massive callus production, a key step for large scale production of PC (Khosroushahi et al. 2011, North et al. 2012). Several attempts have been made to control this browning by using adsorbents and antioxidants (Martinez and Whitaker 1995). Oxidation of phenolic compounds can be controlled by adding polyvinylpyrrolidone (PVP), citric acid (CA), ascorbic acid (AA), glutamine, asparagine, and arginine or doing frequent sub-cultures (Pierik 1997, Rout et al. 2006, Payghamzadeh and Kazemitabar 2011, North et al. 2012). Consequently, this study aimed to investigate the best way to control hazelnut callus browning. With this purpose, we studied the effect of certain media composition, certain additives (PVP, AA, AC and), and cell culture methods were used to overcome callus browning and to reach sufficient growth rate in hazelnut cell cultures.

Hazelnut (Corylus avellana L.) seeds were collected from its natural habitat in north of Iran. The seed shells were removed and the seeds were washed with water and liquid soap to eliminate the lint on the seeds. Then, sterilization of the seeds was performed by sodium hypochlorite $(5.25 \%, \mathrm{~m} / \mathrm{v})$ for $25 \mathrm{~min}$ and then by ethanol $(70 \%, v / v)$ for two min. After rinsing in sterile water (3 $\times 5 \mathrm{~min})$, the sterile cotyledons were cultured as explants in three different plant culture media: MS (Murashige and Skoog 1962), DKW (Driver and Kuniyuki1984), and NRM (Nas and Read 2004) which all were supplemented with $2 \mathrm{mg} \mathrm{dm}^{-3}$ dichlorophenoxyacetic acid (2,4-D), $0.2 \mathrm{mg} \mathrm{dm}^{-3}$ 6-benzylamino purine (BAP), $30 \mathrm{~g} \mathrm{dm}^{-3}$ sucrose, and $8 \mathrm{~g} \mathrm{dm}^{-3}$ agar-agar. The $\mathrm{pH}$ was set to 5.8 . Polyvinylpyrrolidone (PVP, $500 \mathrm{mg} \mathrm{dm}^{-3}$ ) and two antioxidants CA, $500 \mathrm{mg} \mathrm{dm}^{-3}$, and AA, $500 \mathrm{mg} \mathrm{dm}^{-3}$ were added in separated experiment to assess their effect on inhibition of callus browning. The basal media were served as controls. The dishes in all experiments were included conical flask (with a size of $100 \mathrm{~cm}^{3}$ ) containing $30 \mathrm{~cm}^{3}$ of medium and glass jar (with a size of $250 \mathrm{~cm}^{3}$ ) containing $50 \mathrm{~cm}^{3}$ of medium. All cultured were kept at $25 \pm 2{ }^{\circ} \mathrm{C}$ in darkness. Routine sub-cultures of calli were carried out on the same fresh media every $21 \mathrm{~d}$ to obtain homogenous callus.

In newly modified sub-culture method, hazelnut cotyledons were cultured on a solid MS supplemented with $0.2 \mathrm{mg} \mathrm{dm}^{-3} \mathrm{BAP}$ and $2 \mathrm{mg} \mathrm{dm}^{-3}$ 2.4-D. Cultures were incubated at $25 \pm 2{ }^{\circ} \mathrm{C}$ in darkness and the first subculture was performed after $21 \mathrm{~d}$ in the same conditions. For the second sub-culture, the friable and white callus $(3 \mathrm{~g})$ was transferred to $30 \mathrm{~cm}^{3}$ of liquid media with the same composition as mentioned above but without agar in $100-\mathrm{cm}^{3}$ flasks and kept on rotary shaker $(110 \mathrm{rpm})$ at $25 \pm 2{ }^{\circ} \mathrm{C}$. The nutrient solution was refreshed using a sterile syringe every $10 \mathrm{~d}$. Within six weeks (four sub-cultures), the density of cells increased and the suspension was almost homogenize. Then, some cells including the stacked cells on the flask wall, were picked up and transferred to the same solid media again. The immobilized cultures were kept at $25 \pm 2{ }^{\circ} \mathrm{C}$ in darkness. The growth indices or callus browning percentage derived from modified sub-culture method were compared with the result from routine subcultures on solid media after $21 \mathrm{~d}$.

In this study, two growth indices and browning percentage of callus were investigated: 1 ) relative growth rate $(R G R)=(\ln M 1-\ln M 0) / 21$, where M0 was mass of callus in the beginning and M1 was mass of callus after $21 \mathrm{~d}$ (Al-Khayri and Al-Bahrany 2004); 2) relative fresh mass $(\mathrm{RFMG})=\mathrm{M} 1-\mathrm{M} 0 / \mathrm{M} 0$; and 3 ) browning percentage $(\mathrm{BP})=$ brown callus/ total callus $\times 100$.

All experiments were performed in completely randomized design with five independent replications and each dish were considered as one replication. Data were analyzed by $A N O V A$ using the SPSS statistical analysis software (v. 15.0). The statistical significance differences among the mean values were assessed applying the Duncan's multiple-range test $(P<0.05)$.

In the first callus induction culture, $100 \%$ callus production and high growth rate were observed in all media. The effect of medium composition on the growth rate of calli was significant. The best RGR and RFMG were achieved in NMR with AA or CA. The NRM with AA and NRM with PVP resulted in both high RFMG and low callus BP. While, a low BP was observed in the primary callus induction cultures, calli showed more and more browning in subsequent sub-cultures and also decreased RGR and RFMG (Fig. 1). The addition of common browning inhibitors (AA, CA, and PVP) to culture media, had no considerable effect on BP in initial culture and first sub-culture (Fig. 1C). In the first sub-culture, the highest RFMG were reached on NRM with AA or with PVP (Fig. $1 B$ ) and RGR was not affected. During the second sub-culture, when callus browning significantly increased, the BP was found in MS and DKW with all additives. Thus, media composition had significant effect on BP and NRM with AA and NRM with PVP had the lowest amount of brown calli (13.3\%) (Fig. 1C). In addition, the best RGR (0.01 d $\left.\mathrm{d}^{-1}\right)$ and RFMG (0.67) were observed in NRM with PVP and NRM without additives (Fig. $1 A, B$ ). In this experiment, the antioxidants (AA and $\mathrm{CA}$ ) and absorbent (PVP) had significant effect on reducing callus BP during sub-cultures, but they did not increase the growth indices and did not eliminate browning completely.

An innovative sub-culture method was designed and applied to control phenolic secretions and reduction the browning of callus. The calli were transferred from the solid medium to the liquid medium. Cell suspension culture was established during four sub-cultures. Then the hazelnut cells were immobilized in the initial solid media. A callus in solid culture media can be used as a callus storage for later operations. This culture technique significantly increased the callus RGR and RFMG and eliminated callus browning completely (Fig. 2). At a previous experiment, the lowest callus BP was obtained $13.3 \%$ on NRM with PVP in the second sub-culture, 
whereas in a modified sub-culture method, there were no cases of the browning of the callus (Fig. 2C) during subsequent sub-cultures. Consequently, in this method, RGR was $0.67 \mathrm{~d}^{-1}$, and RFMG was 6.5 after transplantation from the liquid medium to the solid medium, and it was almost 10 times higher than on NRM with PVP.

Hazelnut explants that had a great amount of phenolic compounds affect the quality of in vitro tissue culture (Damiano et al. 2004). Changes in the activity of phenolase and peroxidase are the main reasons of callus browning (Mesquita and Lúcia 2013). Increasing phenolase activity and reducing peroxidase activity during the course of oxidation of phenolic compounds are the main causes of callus browning and death of the explants (Jain et al.
2008). Three media (DKW, MS, and NRM) were used for the callus culture of $C$. avellana. Most hazelnut basalsalt formulations are related to DKW and MS. This is suggested that using of similar combination of organic and mineral compounds but in different ratios, may provide successful in vitro culture of different species (Nas and Read 2004). The average component concentration of NRM, except nitrogen, is similar to hazelnut kernels. The amount of nitrogen in NRM is about $15-17 \%$ of that found in hazel kernel (Lott et al. 1995, Nas and Read 2004). So, we examined the effect of three basal media (MS, NRM, DKW), on the callus growth rates and browning. The highest growth rate in the first sub-culture belonged to MS, but in the next sub-culture, NRM had the

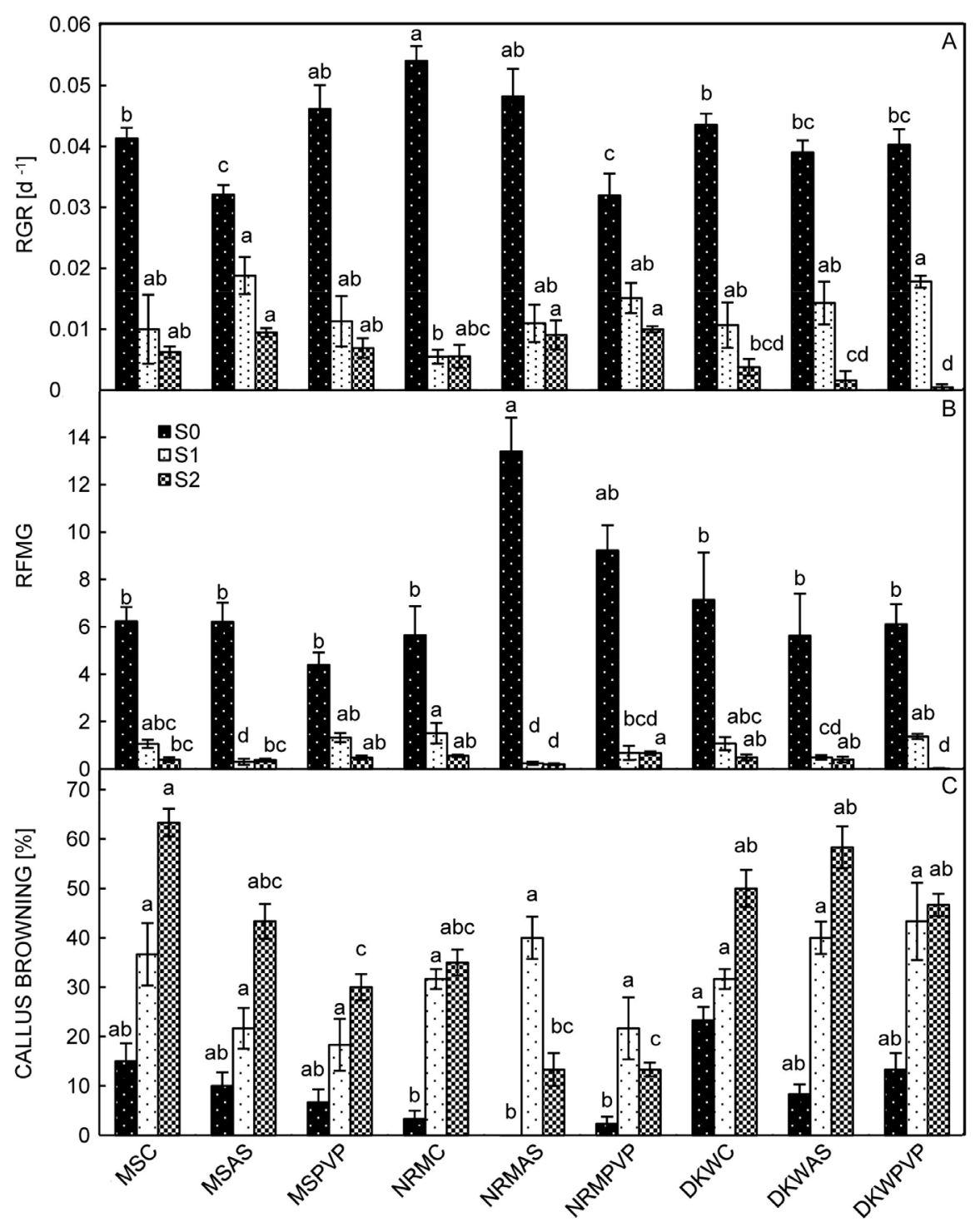

Fig. 1. The effect of different media on a relative growth rate (RGR) $(A)$, relative fresh mass growth (RFMG) $(B)$, and callus browning percentage of Corylus avellana L. (C). MSC - MSC - control Murashige and Skoog (MS) medium without antioxidants and PVP; MSAS - MS with antioxidants; MSPVP - MS with PVP; NRMC - control Nas and Read medium (NRM) without antioxidants and PVP; NRMAS - NRM with antioxidants; NRMPVP - NRM with PVP; DKWC - control Driver and Koniuki medium (DKW) without antioxidants and PVP; DKWAS - DKW with antioxidants; DKWPVP - DKW with PVP. Means \pm SEs, $n=5$. Different letters indicate significant differences $(P<0.05)$ among the treatments (the Duncan's multiple range test). S0, S1, S2 - initial culture, first, and second sub-cultures, respectively. 

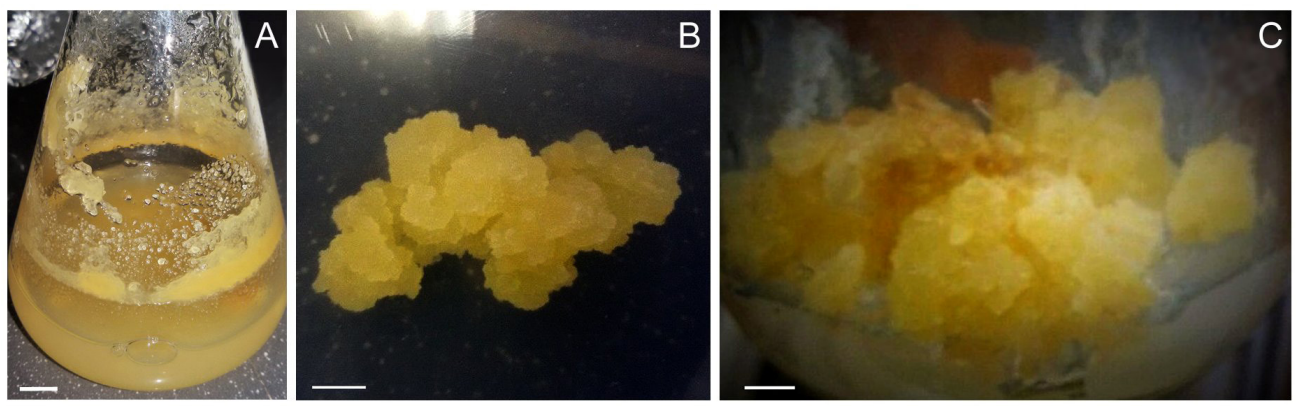

Fig. 2. The steps of cell immobilization to prevent the hazelnut callus browning. $A$ - The cell suspension culture of hazelnut and the callus mass aggregated on the flask wall; $B$ - the growing population of hazelnut cells after being retransferred to a solid medium after a month; $C$ - a massive friable callus obtained from a modified sub-culture system after two months. Scale bars equal $70 \mathrm{~mm}(A$ and $C)$ and $30 \mathrm{~mm}(B)$.

highest growth rate. Getting the mass of white and flexible callus through the successive sub-cultures, NRM was best between the media used. As concern macroelements, Nas and Read (2004) adjusted $\mathrm{MgSO}_{4}$ at $1600 \mathrm{mg} \mathrm{dm}^{-3}$ and $\mathrm{KH}_{2} \mathrm{PO}_{4}$ at $1300 \mathrm{mg} \mathrm{dm}^{-3}$ that were much higher than in $\mathrm{MS}$ and DKW. $\mathrm{K}_{2} \mathrm{SO}_{4}$ is absent in NRM and MS. The amount of $\mathrm{H}_{3} \mathrm{BO}_{3}$ in NRM was set similar to MS that is a little higher than in DKW (Nas and Read 2004, Hand 2013). In NRM, the amount of myo-inositol is twice more than in MS and DKW. Nas and Read (2004) used $100 \mathrm{mg}$ $\mathrm{dm}^{-3}$ of sequestrene $138 \mathrm{Fe}$ as Fe resource in NRM. Some previous studies demonstrated that MS medium is not suitable enough for in vitro hazelnut culture (Al Kaï et al. 1984, Diaz-Sala et al. 1990). The NRM also has some amount of vitamin $\mathrm{C}$ (ascorbic acid), which can make it a good candidate for cultures, which have a browning problem. In the second sub-culture, NRM with PVP had the highest growth rate and NRM with PVP and NRM with AA had the highest prevention of callus browning that is due to the present of antioxidant such as AA and CA as well as phenolic absorbent compounds like PVP. PVP and active carbon were successful in reducing oxidation that were related to stress and improving of regeneration of explants. They are usually used to prevent from tissue blackening and auto oxidation (Toth et al. 1994, Thomas 2008, Uchendu et al. 2011, Reed et al. 2012). PVP and CA + AA in combination with NRM reduced callus browning significantly, but not eliminated it.

The PC is one of the most effective anticancer drugs that were found in Corylus, but its amount is limited in this source (Gallego et al. 2017). It have fast growing callus in the initial steps of culture but it is heavily affected by browning. Therefore, we were looking for a method that causes $100 \%$ increasing in vitro callus initiation and can prevent callus browning. Although, hazelnut callus induction was successful, callus browning was increased through frequent sub-cultures. After first or eventually second sub-culture, white and friable callus was transfer to liquid medium, because liquid medium reduces the phenolic secretion (Benson 2000). The liquid medium has many advantages (Benson 2000): replication is high because the contact between the explants and media is higher than in solid media and the access to water and nutrients more sophisticated (Pierik 1997, Mbiyu et al. 2012). The conditions of culture are uniform and sub-culture is possible without changing the containers (Berthouly and Etienne 2005). Cultivation of cell suspension provides a versatile tool for different research and even drug metabolites can be find in cell suspension (Preil 2005, Wilken et al. 2005, Al-Khayri 2012). The studies of Phoenix dactylifera indicated that cell suspension produced high and fresh mass callus in a less time (Ibraheem et al. 2013). So, novel change in routine sub-culture method performed to use liquid media have advantages and eliminate the browning of callus in C. avellana callus culture. The callus derived from this method was completely white and friable with fast growth rate. Fast-growing callus in solid medium would be a good resource for later studies.

\section{References}

Abbasin, Z. Zamani, S. Movahedi, S. Khaksar, G. Tabatabaei, B.S.: In vitro micropropagation of yew (Taxus baccata) and production of plantlets. - Biotechnology 9: 48-54, 2010.

Al Kaï, H. Salesses, G. Mouras, A.: In vitro multiplication of hazel (Corylus avellana L.). - Agronomie 4: 399-402, 1984.

Al-Khayri, J.M.: Determination of the date palm cell suspension growth curve, optimum plating efficiency, and influence of liquid medium on somatic embryogenesis. - Emir. J. Food Agr. 24: 444-455, 2012.

Al-Khayri, J. Al-Bahrany, A.: Growth, water content, and proline accumulation in drought-stressed callus of date palm. - Biol. Plant. 48: 105-108, 2004.

Bedi, Y. Ogra, R. Kiran, K. Kaul, B. Kapil, R.: Yew (Taxus spp.). A new look on utilization, cultivation and conservation. - In: Handa, S.S., Kaul, M.K. (ed.): Supplement to Cultivation and Utilization of Medicinal Plants. Pp. 443-456. Regional Research Laboratory, Jammu - Tawi 1996.

Benson, E.E.: Do free radicals have a role in plant tissue culture recalcitrance? - In vitro cell. dev. Biol. Plant. 36: 163-170, 2000.

Berthouly, M. Etienne, H.: Temporary immersion system: a new concept for use liquid medium in mass propagation. - In: Hvoslef-Eide, A.K., Preil, W. (ed.): Liquid Culture Systems for In Vitro Plant Propagation. Pp. 165-195. Springer, Dordrecht 2005.

Bestoso, F. Ottaggio, L. Armirotti, A. Balbi, A. Damonte, G. Degan, P. Mazzei, M. Cavalli, F. Ledda, B. Miele, M.: In vitro cell cultures obtained from different explants of Corylus avellana produce taxol and taxanes. - BMC Biotechnol. 6: 1-11, 2006. 
Cronquist, A. Takhtadzhian, A.L.: An Integrated System of Cassification of Fowering Plants. - Columbia University Press, New York 1981.

Damiano, C. Catenaro, E. Giovinazzi, J. Frattarelli, A. Caboni E.: Micropropagation of hazelnut (Corylus avellana L.). In: Tous, J., Rovira, M., Romero, A. (ed.): VI International Congress on Hazelnut. Pp. 221-226. Acta Hort., Rome 2005.

Diaz-Sala, C. Rey, M. Rodriguez, R.: In vitro establishment of a cycloclonal chain from nodal segments and apical buds of adult hazel (Corylus avellana L.). - Plant Cell Tissue Organ Cult. 23: 151-157, 1990.

Driver, J. A. Kuniyuki, A. H.: In vitro propagation of Paradox walnut rootstock. - HortScience 19: 507-509, 1984.

Gallego, A. Malik, S. Yousefzadi, M. Makhzoum, A. Tremouillaux-Guiller, J. Bonfill, M.: Taxol from Corylus avellana: paving the way for a new source of this anti-cancer drug. - Plant Cell Tissue Organ Cult. 129: 1-16, 2017.

Georgiev, M.I. Weber, J. Maciuk, A.: Bioprocessing of plant cell cultures for mass production of targeted compounds. - Appl. Microbiol. Biotechnol. 83: 809-823, 2009.

Hand, C.R.: Improving initiation and mineral nutrition for hazelnut (Corylus avellana) micropropagation. - Thesis. Oregon State University, Corvallis 2013.

Hoffman, A. Khan, W. Worapong, J. Strobel, G. Griffin, D. Arbogast, B. Barofsky, D. Boone, R.B. Ning, L. Zheng, P.: Bioprospecting for taxol in angiosperm plant extracts using high performance liquid chromatography thermospray mass spectrometry to detect the anticancer agent and its related metabolites in filbert trees. - Spectroscopy 13: 22-32, 1998.

Hoffman, A. Shahidi, F.: Paclitaxel and other taxanes in hazelnut. - J. Funct. Foods. 1: 33-37, 2009

Hussain, M.S. Fareed, S. Saba Ansari, M. Rahman, A. Ahmad, I.Z. Saeed, M.: Current approaches toward production of secondary plant metabolites. - J. Pharm. Bioallied Sci. 4: 10, 2012.

Ibraheem, Y. Pinker, I. Böhme, M.: A comparative study between solid and liquid cultures relative to callus growth and somatic embryo formation in date palm (Phoenix dactylifera L.) cv. Zaghlool. - Emir. J. Food Agr. 24: 889-898, 2013.

Jain, S. Kharya, M. Nayak, S. Barik, R.: Effect of antioxidants on callus browning of Glycyrrhiza glabra. - J. Natur. 8: 4447, 2008

Khosroushahi, A.Y. Naderi-Manesh, H. Simonsen, H.T.: Effect of antioxidants and carbohydrates in callus cultures of Taxus brevifolia: evaluation of browning, callus growth, total phenolics and paclitaxel production. - BioImpacts 1: 37, 2011.

Loewus, F.A. Murthy, P.P.: Myo-inositol metabolism in plants. Plant Sci. 150: 1-19, 2000.

Lott, J.N. Greenwood, J.S. Batten, G.D.: Mechanisms and regulation of mineral nutrient storage during seed development. - In: Kigel, J., Gallili, G. (ed.): Seed Development and Germination. Pp. 215-235. Marcel Dekker, New York 1995.

Martinez, M.V. Whitaker, J.R.: The biochemistry and control of enzymatic browning. - Trends Food Sci Technol. 6: 195-200, 1995.

Mbiyu, M. Muthoni, J. Kabira, J. Muchira, C. Pwaipwai, P. Ngaruiya, J. Onditi, J. Otieno, S.: Comparing liquid and solid media on the growth of plantlets from three Kenyan potato cultivars. - Amer. J. exp. Agr. 2: 81, 2012.

Mesquita, V. Lúcia, V.: Enzymatic browning. - In: Eskin, N.A.M. Shahidi, F. (ed.): Biochemistry of Foods. ${ }^{\text {rd }}$ Ed. Pp. 387-418. Academic Press, San Diego 2013.

Miele, M. Mumot, A.M. Zappa, A. Romano, P. Ottaggio, L.: Hazel and other sources of paclitaxel and related compounds.
- Phytochem. Rev. 11: 211-225, 2012.

Molnar, T.: Expansion of hazelnut research in North America. Fao Newslett. 15: 33-37, 2011.

Murashige, T. Skoog, F.: A revised medium for rapid growth and bio assays with tobacco tissue cultures. - Physiol. Plant. 15: 473-497, 1962.

Nas, M.N. Read, P.E.: A hypothesis for the development of a defined tissue culture medium of higher plants and micropropagation of hazelnuts. - Sci. Hort. 101: 189-200, 2004.

North, J. Ndakidemi, P. Laubscher, C.: Effects of antioxidants, plant growth regulators and wounding on phenolic compound excretion during micropropagation of Strelitzia reginae. - Int. J. phys. Sci. 7: 638-646, 2012.

Payghamzadeh, K. Kazemitabar, S. K.: In vitro propagation of walnut. - Afr. J. Biotechnol. 10: 290-311, 2011.

Pierik, R.L.M.: In Vitro Culture of Higher Plants. - Springer, Dordrecht 1997.

Preil, W.: General introduction: a personal reflection on the use of liquid media for in vitro culture. - In: Hvoslef-Eide, A.K. Preil, W. (ed.): Liquid Culture Systems for in Vitro Plant Propagation. Pp. 1-18. Springer, Dordrecht 2005.

Qaderi, A. Omidi, M., Etminan, A. Oladzad, A., Ebrahimi, C., Dehghani, M.R., Mehrafarin, A.: Hazel (Corylus avellana L.) as a new source of taxol and taxanes. - J. med. Plants 1: 6677, 2012.

Rahpeyma, S.A. Moieni, A. Jalali-Javaran, M.: Enhancement of paclitaxel content in induced tetraploid Corylus avellana L. cell suspension culture with regulating the expression of genes in paclitaxel biosynthetic pathway. - Acta Physiol. Plant. 39: 1-8, 2017.

Reed, B.M. Uchendu, E. Normah M.: Are anti-oxidants effective for reducing oxidative stress during cryopreservation. - In: Proceedings of the $1^{\text {st }}$ International Symposium for In Vitro Conservation and Cryopreservation. Vol. 1. Pp. 1-8. Tepatitlan de Morolos, Jalisco 2012.

Rout, G. Mohapatra, A. Jain, S.M.: Tissue culture of ornamental pot plant: a critical review on present scenario and future prospects. - Biotechnol. Adv. 24: 531-560, 2006.

Salehi, M. Moieni, A. Safaie, N. Farhadi, S.: Elicitors derived from endophytic fungi Chaetomium globosum and Paraconiothyrium brasiliense enhance paclitaxel production in Corylus avellana cell suspension culture. - Plant Cell Tissue Organ Cult. 8: 1-11, 2018.

Sugano, N. Iwata, R. Nishi, A.: Formation of phenolic acid in carrot cells in suspension cultures. - Phytochemistry 14: 1205-1207, 1975.

Thomas, T.D.: The role of activated charcoal in plant tissue culture. - Biotechnol. Adv. 26: 618-631, 2008.

Toth, K., Haapala, T., Hohtola, A.: Alleviation of browning in oak explants by chemical pretreatments. - Biol. Plant. 36: 511517, 1994.

Uchendu, E.E. Paliyath, G. Brown, D.C. Saxena, P.K.: In vitro propagation of North American ginseng (Panax quinquefolius L.). - In Vitro cell. dev. Biol. Plant. 47: 710-718, 2011.

Wilken, D. González, E.J. Hohe, A. Jordan, M. Kosky, R.G. Hirschmann, G.S. Gerth A.: Comparison of secondary plant metabolite production in cell suspension, callus culture and temporary immersion system. - In: Hvoslef-Eide, A.K., Preil, W. (ed.): Liquid Culture Systems for in Vitro Plant Propagation. Pp. 525-537. Springer, Dordrecht 2005.

Yu, X. Reed, B.M.: A micropropagation system for hazelnuts (Corylus species). - HortScience 30: 120-123, 1995. 